\title{
Evaluation of the backoff procedure of Homeplug MAC vs. DCF
}

\author{
Cristina Cano and David Malone \\ Hamilton Institute \\ National University of Ireland, Maynooth \\ Co. Kildare, Ireland \\ Email: \{cristina.cano,david.malone $\} @$ nuim.ie
}

\begin{abstract}
The standardized Medium Access Control (MAC) protocols for Power Line Communication (PLC) networks (Homeplug and IEEE 1901) are based on the Distributed Coordination Function (DCF) defined for IEEE 802.11. However, the backoff procedure is modified with the goal of decreasing the collision probability. In this work, the backoff procedure of PLC MAC protocols is compared to DCF in different traffic conditions and scenarios, including scenarios with all nodes in coverage range as well as topologies with hidden and exposed terminal problems. The goal is to demonstrate and quantify the pros and cons of each approach in each particular case. Results show that the modified backoff procedure of the Homeplug MAC reduces the collision probability when there is high contention. However, the performance is not always improved compared to DCF. Moreover, when Homeplug provides better performance than the vanilla DCF, we show that the DCF can be easily tuned to achieve similar gains.
\end{abstract}

\section{INTRODUCTION}

Protocols designed for Power Line Communication (PLC) networks have many characteristics in common with protocols for Wireless Local Area Networks (WLANs) mainly due to the similarities of PLC and wireless channels. These similarities include propagation impairments (although the nature of the impairments to the PLC channel are different) and problems like hidden/exposed terminals. That is the reason why the Medium Access Control (MAC) layer of the Homeplug standard [1] (also the same defined as IEEE 1901 [2]) is based on the Distributed Coordination Function (DCF) defined in the IEEE 802.11 [3] standard for WLANs. One of the main differences between them is the modified backoff procedure implemented in Homeplug that aims at further reducing the collision probability by decreasing transmission attempts when high traffic contention is inferred on the channel.

The behaviour of the DCF has been extensively analysed in the past years and efforts have been made by the research community to improve its performance in various network conditions and configurations. In contrast, the study of the performance benefits of Homeplug MAC vs. DCF has not obtained much attention yet. In fact, as far as the authors know, there are only three articles that make an effort to compare the performance of both protocols, as follows. In [4], the Homeplug MAC is compared in saturation conditions and when all nodes are in mutual coverage range, the conclusion drawn is that the Homeplug MAC works better under these considerations and large packet sizes. A comparison is also made in [5], where a flow-level evaluation is done in a multihop topology showing that the throughput in the scenarios evaluated is improved with Homeplug. Short-term fairness is investigated in [6] in a scenario with all nodes in mutual coverage range and saturated conditions and it is concluded that the Homeplug MAC is fairer when the number of contending nodes is higher than 15 . Therefore, there is still much work to do to fully demonstrate and quantify the performance improvement (if any) of the Homeplug MAC compared to DCF in different scenarios and traffic conditions.

This work takes a further step in evaluating the conditions and scenarios under which the Homeplug MAC provides higher performance than the DCF protocol. For that purpose, both protocols are studied with comparable parameter configurations so as to only evaluate the modification of the backoff procedure but not the specific parameter settings. Furthermore, we also show how the DCF can be easily configured to obtain similar performance as the Homeplug MAC when the latter outperforms the regular DCF. This issue is of relevance when the additional complexity of the Homeplug MAC is considered and especially when evaluating the Homeplug MAC as a refinement of DCF [4].

This work is divided as follows. Section II presents an overview of the Homeplug and DCF MAC protocols. Next, Section III discusses how the DCF can be modified to achieve similar performance gains as the Homeplug MAC. Then, the methodology and results are described in Section IV. Finally, some conclusions and future research are outlined.

\section{HomePlug AND DCF RANDOM BACKOFF PROCEDURES}

Using DCF, each time a node has a new packet to transmit, the backoff stage $(i \in[0, m])$ is initialized to 0 and a random backoff is selected among $\left[0, W_{0}-1\right]$. Both, the initial contention window $\left(W_{0}\right)$ and the maximum backoff stage $(m)$ are tunable parameters. The backoff countdown is frozen when activity is detected on the channel and restarted when the medium becomes idle again. The packet is actually transmitted when the backoff countdown expires. If an acknowledgement is received, the packet is considered successfully transmitted. Otherwise, the node starts the retransmission procedure: the new backoff stage changes to $i=\min (i+1, m)$ and a new random backoff is selected among $\left[0, W_{i}-1\right]$, being $W_{i}$ the 


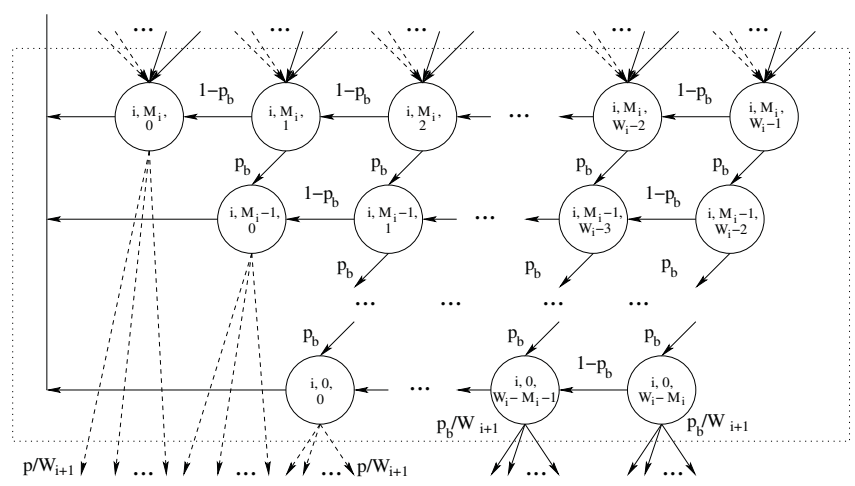

Fig. 1. Markov Chain Model in Backoff Stage $i$ of the Homeplug MAC [9]

contention window of the new backoff stage. The value of $W_{i}$ is $W_{i}=2^{i} W_{0}$. This is the reason why the backoff procedure in DCF is known as Binary Exponential Backoff (BEB).

The backoff procedure in the Homeplug MAC follows the same general approach as the one used in DCF. However, a new counter, called the Deferral Counter (DC), is introduced. This counter is initialized at each backoff stage to $M_{i}$ (all $M_{i}$ are tunable parameters) and decreased by one after overhearing a data packet or a collision ${ }^{1}$. If a new packet or a collision are overheard and the value of the DC is equal to zero, the node acts as if a collision had happened: the backoff stage is increased if it has not yet reached its maximum value and a new random backoff is selected among $\left[0, W_{i}-1\right]$ (all $W_{i}$ are tunable parameters in Homeplug). Observe that this procedure aims to avoid collisions when high contention is inferred by decreasing the aggressiveness of transmission attempts.

Both backoff procedures have been mathematically modeled in the literature. Bianchi [7] defined a simple mathematical model of the DCF by modeling each node as a Markov Chain and considering the collision probability independent of the backoff stage. This analytical model has been further extended by several authors, for instance, in [8], the analysis is extended to compute the channel access delay. The Homeplug MAC has also been modeled following the Bianchi approach, the most well-know, although complex, analytical model is the one presented and validated in [9]. Fig. 1 shows the part of the Markov Chain corresponding to a given backoff stage with $i \in[1, m-1]$. It can be observed how the DC counter is decreased when the channel is detected busy (that happens with $p_{b}$ probability) and how the new backoff stage is increased when the DC counter reaches zero and a new packet or collision are overheard. The similarities with the DCF backoff procedure and analytical model in [7] can also be noted.

\section{DCF WITH $k$-ARY EXPONENTIAL BACKOFF}

While the Homeplug MAC also reacts to overheard packets to reduce the transmission attempt probability, DCF only reacts

${ }^{1}$ Carrier sense is activated in Homeplug either when a preamble or a priority resolution symbol are detected. Therefore, in both cases (successful reception of a packet and collision overheard) the DC is decreased. to collisions. One possibility for tuning the DCF to perform similarly to the Homeplug MAC is to more rapidly increase the contention window at each backoff stage. As explained in the previous section, the DCF doubles the contention window at every backoff stage (after each collision). Therefore, we propose replacing the $\mathrm{BEB}$ of DCF to a $k$-ary exponential backoff with the goal of decreasing the collision probability when the traffic load increases. Therefore, the contention window at backoff stage $i$ will be computed as $W_{i}=k^{i} W_{0}$, where both $W_{0}$ and $k$ are tunable parameters. This extension of the DCF backoff procedure was already proposed in [10] for traffic differentiation purposes.

To analytically model the $k$-ary exponential backoff is straightforward by using the Bianchi [7] and Chatzimisios et al. [8] expressions and modifying $W_{i}$ to consider the new increase factor $k$. The new transmission probability $(\tau)$ and average channel access delay $(E[X])$ can then be computed as shown in Eqs. 1 and 2, respectively.

$$
\begin{aligned}
\tau & =\frac{2(1-k p)}{(1-k p)+W_{0}(1-p)+p W_{0}(k p)^{m}(1-k)} . \\
E[X] & =\frac{\alpha\left(p W_{0}\left[(1-k)(k p)^{m}-1\right]-k p+W_{0}+1\right)}{2(1-k p)(1-p)} .
\end{aligned}
$$

where $p$ denotes the conditional collision probability and $\alpha$ is the average slot duration, refer to [7] and [8] for details.

\section{Performance Evaluation}

In this section, the performance evaluation of the three approaches: DCF with BEB $(k=2)$, DCF with $k$-ary $(k>2)$ exponential backoff and Homeplug, are evaluated in different scenarios and traffic conditions. When saturated conditions are considered, the mathematical models in [7], [8] and [9] as well as our extension in Eqs. 1 and 2 are used. For both saturated and unsaturated cases, simulations are performed as in the case of Homeplug they have been found more computationally efficient than the analysis. A custom simulator based on the SENSE framework [11] has been used. If not otherwise specified, simulation results show average values (the confidence intervals are too small to be shown) of 5 simulation runs. The minimum simulation time is $10,000 \mathrm{~s}$.

The assumptions and considerations taken into account are as in [7], [8] and [9]: infinite, or large enough to be considered infinite, queue length and retry limit, exponentially distributed interarrival of packets and ideal channel conditions. Moreover, no traffic differentiation is considered as the goal is to focus on the evaluation of the different random backoff procedures.

To obtain results not affected by the different parameter settings, equivalent parameters must be considered. The Homeplug 1.0 parameters, shown in Table I have been used for both Homeplug and DCF. Contention parameters in DCF have been set to $W_{0}=8$ and $m=3$, while in Homeplug these parameters are configured as $W=\langle 8,16,32,64\rangle$ with $M=\langle 0,1,3,15\rangle$. Observe that the contention windows at each backoff stage are the same. Therefore, this configuration 


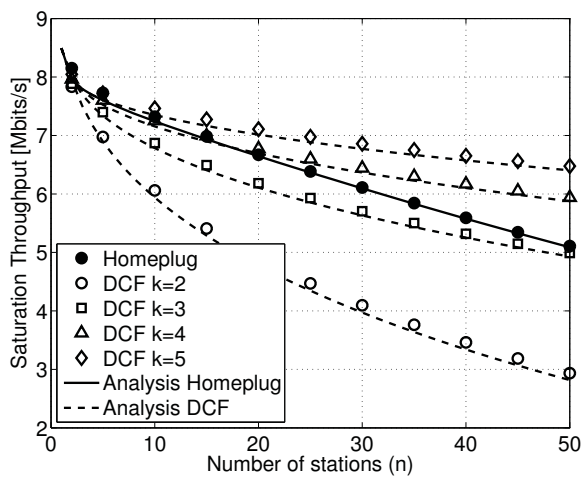

(a) Throughput

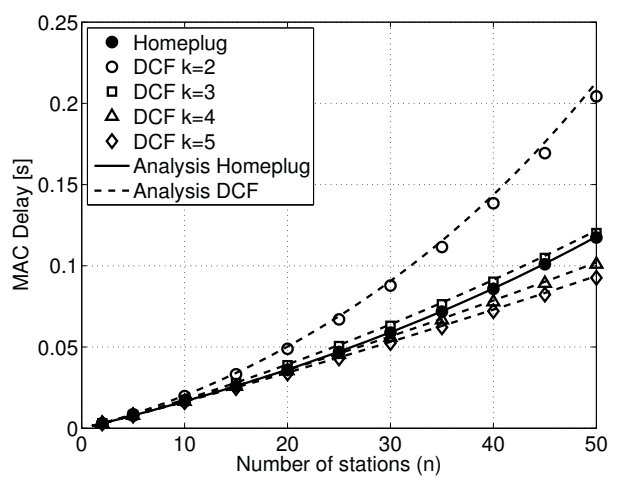

(b) Channel access delay

Fig. 2. Performance metrics of Homeplug and DCF in a fully connected network with homogeneous nodes and in saturated conditions.

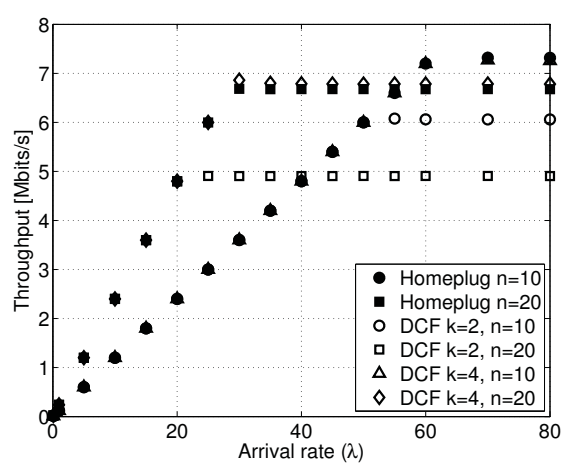

(a) Throughput

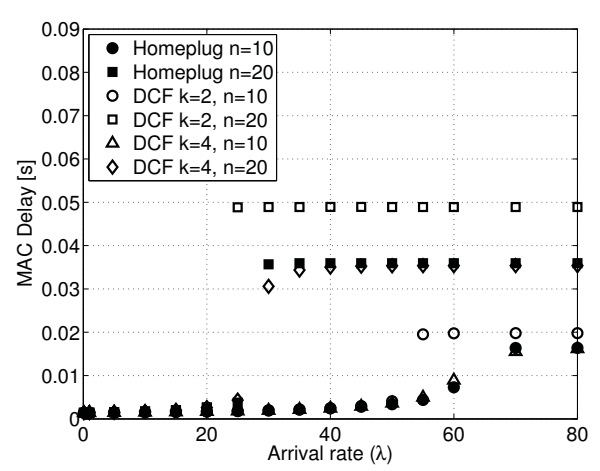

(b) Channel access delay

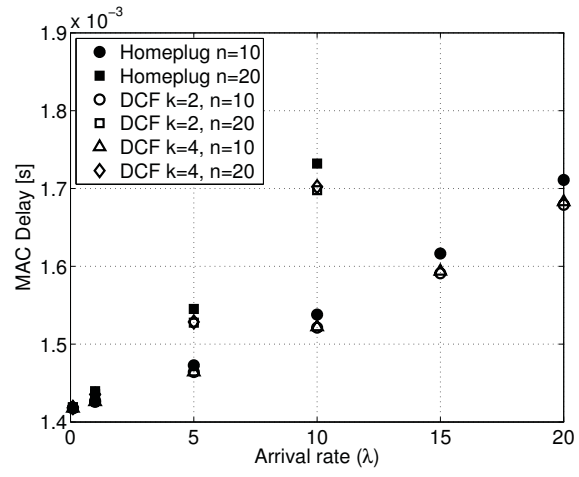

(c) Channel access delay for $\lambda<20$ packets/s

Fig. 3. Performance metrics of Homeplug and DCF in a fully connected network with homogeneous nodes and in unsaturated conditions.

TABLE I

PARAMETERS

\begin{tabular}{|c||c|}
\hline Parameter & Value in Homeplug 1.0 \\
\hline Slot time $(\sigma)$ & $35.84 \mu \mathrm{s}$ \\
\hline Data rate $(R)$ & $14 \mathrm{Mbps}$ \\
\hline Frame transmission time $\left(T_{\text {fra }}\right)$ & $1153.5 \mu \mathrm{s}$ \\
\hline ACK transmission time $\left(T_{\text {ack }}\right)$ & $72 \mu \mathrm{s}$ \\
\hline Data-ACK interframe space $($ RIFS $/$ SIFS) & $26 \mu \mathrm{s}$ \\
\hline Contention interframe space $($ CIFS $/$ DIFS) & $35.84 \mu \mathrm{s}$ \\
\hline
\end{tabular}

will allow us to specifically evaluate the effect of the DC. The starting values $M$ are these recommended by the standard.

The transmission time duration considered corresponds to 1500 bytes in Homeplug 1.0, that is the maximum payload and therefore, it represents the worst case for the DCF as the cost of collisions is the highest. A comparison of the performance using different packet sizes was already performed in [4].

\section{A. Fully Connected Network and Homogeneous Nodes}

The first scenario evaluated is a fully connected network in which all $n$ nodes are in mutual coverage range. Homogeneous nodes are also considered, i.e., all nodes use the same parameters and generate packets at the same rate.

Throughput and delay in saturated conditions are shown in Fig. 2. It can be observed that results are comparable when a small number of nodes are contending. Conversely, when the number of nodes increases and therefore, the collision probability also increases, the difference among the BEB DCF (DCF with $k=2$ ) and the Homeplug is notable (with $n=50$ the throughput increase is of approximately the $65 \%$ as shown in Fig. 2(a)). However, note that increasing the value of $k$ in DCF improves the performance both in terms of throughput and delay (Fig. 2(b)) and that the results obtained are comparable when $k=4$. The higher the value of $k$, the less the aggressiveness in transmission attempts and therefore, the higher the performance as traffic contention increases.

It is important to emphasize that analysing the system in saturation is not enough to draw conclusions about the performance of the different protocols. The unsaturated results for $n=10$ and $n=20$ are shown in Fig. 3. Observe how the saturation point for DCF with $k=2$ is lower than for Homeplug or DCF with $k=4$ (Fig. 3(a)). A similar observation can be made for delay (Fig. 3(b)). An important result is also obtained for low traffic loads (Fig. 3(c)). When $\lambda<20$ packets/s, the channel access delay is comparable for all three evaluated approaches with Homeplug MAC showing a slightly longer delay. This is caused by the higher probability of overhearing a packet instead of facing a collision, which unnecessarily increases the average waiting time. 


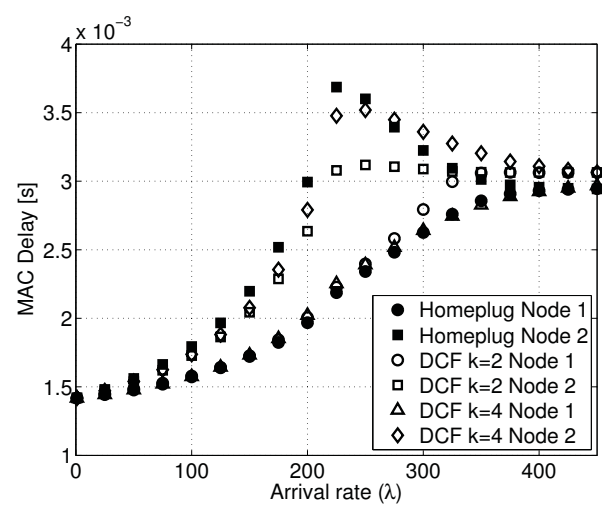

(a) Channel access delay, $\lambda_{n_{1}}=2 \lambda$

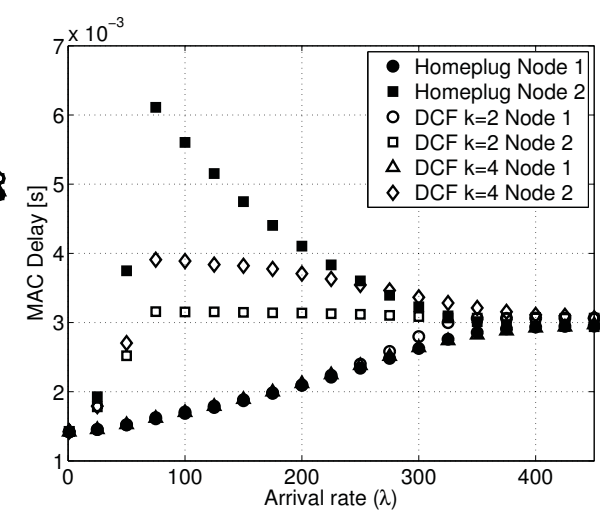

(b) Channel access delay, $\lambda_{n_{1}}=10 \lambda$

Fig. 4. Performance metrics of Homeplug and DCF in a fully connected network with heterogeneous nodes.

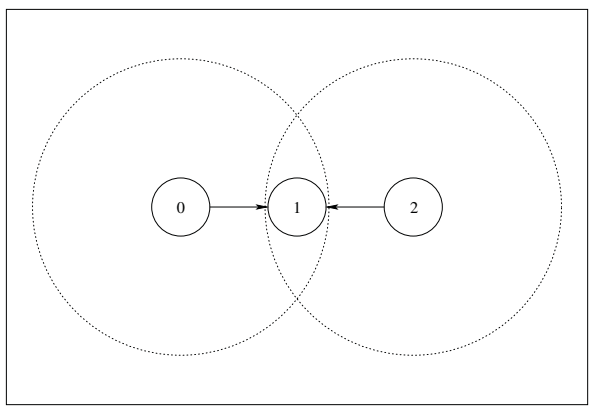

(a) Hidden terminal scenario

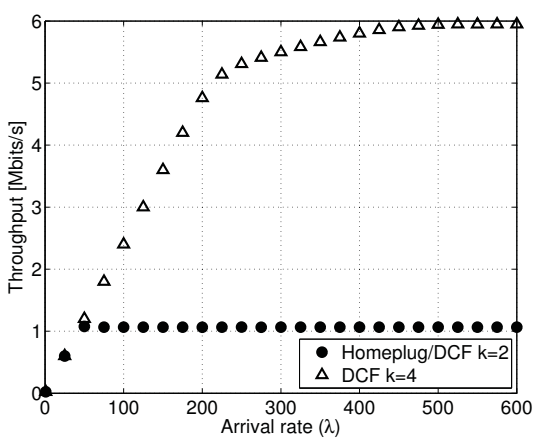

(b) Throughput

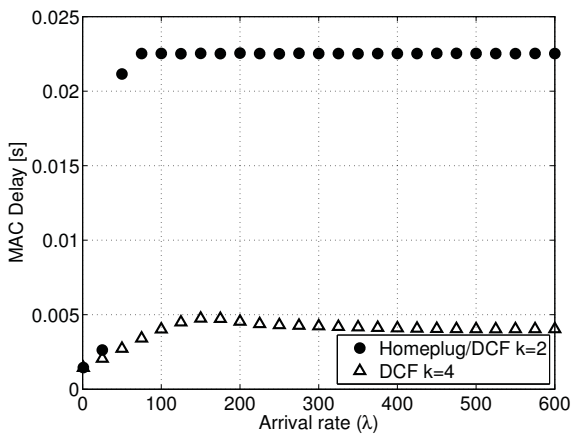

(c) Channel access delay

Fig. 5. Topology and performance metrics of Homeplug and DCF in a hidden terminal scenario.

\section{B. Fully Connected Network and Heterogeneous Nodes}

In this scenario we evaluate the long-term fairness in unsaturated conditions for a fully connected network in which nodes generate packets at different rates. For that purpose, we set $n=2$ and increase the traffic generation rate of node 1 .

Channel access delay results for $\lambda_{n_{1}}=2 \lambda$ and $\lambda_{n_{1}}=10 \lambda$ are shown in Fig. 4(a) and Fig. 4(b) respectively. When saturation is reached, the delays of node 1 and node 2 become equal for each backoff mechanism and the difference between each approach appears due to the number of nodes contending for the channel (as evaluated in the last subsection). The channel access delay of node 1 before saturation is smaller than the one observed for node 2 in all three cases (DCF with $k=2$, DCF with $k=4$ and Homeplug). This effect is caused by the smaller collision probability faced by node 1. This difference is more noticeable in DCF using $k=4$ than $k=2$ since in the former the backoff waiting time is, on average, longer after a collision. But, observe that, using Homeplug, node 2 is more heavily penalised. In Homeplug, on average, the backoff waiting time is increased for node 2 due to the more frequent overhearing of packets from node 1 . The largest difference is found when $\lambda_{n_{1}}=10 \lambda$ and $\lambda=75$ packets/s, where the delay with the Homeplug MAC for node 2 is twice the delay obtained for DCF with $k=2$ while the delay obtained for node 1 is comparable.

\section{Hidden Terminal Problem}

In this subsection we analyse the three approaches in a classical toy scenario in wireless and PLC networks, that is, when there are nodes that are not able to sense each other and they transmit to a common receiver. We have evaluate the case in which two hidden nodes transmit to a third, common neighbour (see Fig. 5(a)).

Total throughput and channel access delay are shown in Fig. 5(b) and Fig. 5(c) respectively. Note that, there is no overhearing in this case as the 2 hidden terminals, that are the only transmitting nodes, cannot overhear each others transmissions. Consequently, given that the setting parameters are the same for both, Homeplug MAC and DCF with $k=2$ result in exactly the same performance. This is the reason why only Homeplug and DCF with $k=4$ performance results are shown in Figs. 5(b) and 5(c). Increasing $k$ increases the probability that one of the hidden nodes is able to completely transmit a packet while the other is waiting for the backoff countdown to expire. Observe that the saturated throughput improvement using DCF with $k=4$ instead of $k=2$ and Homeplug is considerable (approximately a $500 \%$ increase). 


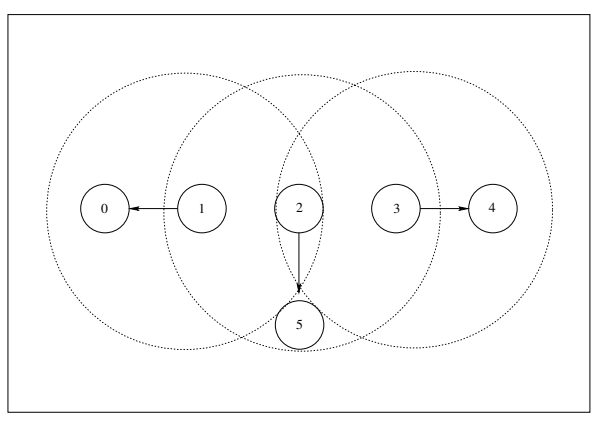

(a) Node-in-the-middle scenario

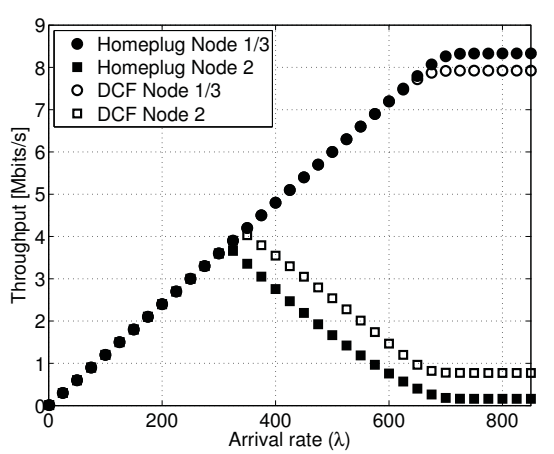

(b) Throughput

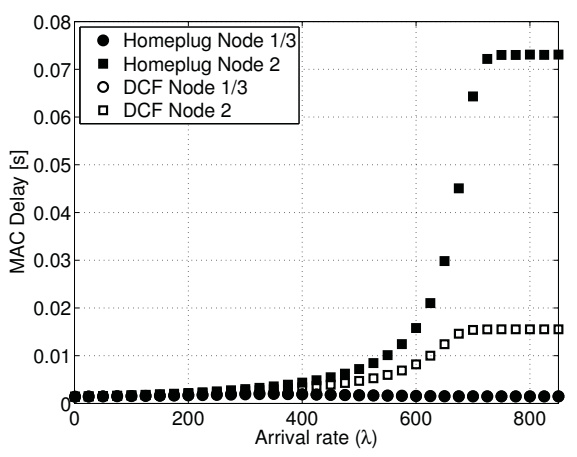

(c) Channel access delay

Fig. 6. Topology and performance metrics of Homeplug and DCF in a node-in-the-middle scenario.

\section{Node-in-the-Middle Problem}

In this case we also consider a well-known problem in which a node overhears packets from two other nodes that are not in coverage range and all of them transmit to receivers that cannot overhear any of the others transmissions (see Fig. 6(a)). This configuration results in receivers able to successfully decode all packets generated by the transmitters (there are no collisions). This is a particular case of the exposed terminal problem. However, it is even more problematic for the node that overhears packets from the others (the node-in-themiddle). Being able to overhear packets from two transmitters that are not in coverage range translates to a low probability to acquire the channel for transmission.

The results of 20 simulation runs for the node-in-themiddle (node 2) and the per-node throughput and delay for the hidden nodes (nodes 1/3) are shown in Fig. 6 (DCF Node 1/3 markers are hidden by Homeplug Node 1/3 in Fig. 6(c)). In this particular scenario, the value of $k$ in DCF does not affect the results because no collisions occur at the receivers. Therefore, DCF does not increase the contention window. The differences are then only due to the effect of the deferral counter. For both approaches, the node-in-the-middle is considerably disadvantaged when the traffic load increases if compared to nodes 1/3. However, in Homeplug, the nodein-the-middle finds it even harder to transmit since, in addition to the low probability of detecting the channel empty, it waits, on average, longer in backoff due to the effect of the deferral counter.

\section{Conclusions}

The backoff procedures of the Homeplug MAC and DCF have been analysed in different scenarios and traffic conditions with the goal of quantifying which approach works better in each case. The modification of the backoff procedure proposed in Homeplug MAC does reduce the collision probability when there is high contention. However, this modification does not always provide better performance than the DCF, especially considering heterogeneous and exposed terminal scenarios. Furthermore, it has been demonstrated that replacing the Homeplug backoff procedure by DCF with $k$-ary exponential backoff provides similar gains. This is of relevance considering the added complexity of the Homeplug MAC in terms of implementation. Additionally, the DCF with $k$-ary exponential backoff shows higher performance in hidden terminal scenarios compared to both Homeplug and DCF with BEB.

Further evaluation to obtain more insight on the comparison of both MAC layers is necessary. Research lines of interest are the consideration of traffic differentiation capabilities (Homeplug vs. the IEEE 802.11e EDCA specification), optimal tuning of the contention parameters in both approaches, as well as the evaluation in scenarios affected by interference.

\section{ACKNOWLEDGMENTS}

This work has been partially supported by the Science Foundation Ireland grant 08/SRC/I1403.

\section{REFERENCES}

[1] Homeplug Powerline Alliance, "Homeplug 1.0 Specification," 2001.

[2] IEEE Std 1901, "Standard for Broadband over Power Line Networks: Medium Access Control and Physical Layer Specifications," ANSI/IEEE Std 1901, 2010.

[3] IEEE Std 802.11, "Wireless LAN Medium Access Control (MAC) and Physical Layer (PHY) Specifications," ANSI/IEEE Std 802.11, 1999 Edition (Revised 2007)

[4] P. B. Velloso, M. Elias, M. Campista, D. D. O. Cunha, L. H. M. K Costa, and O. C. M. B. Duarte, "Analyzing the performance of wireless local area networks with an improved collision avoidance mechanism," Technical Report.

[5] L. Jian and S. Qiang, "The study on the performance of backoff algorithms in multihop power line communication networks," in Proceedings of the Third International Conference on Measuring Technology and Mechatronics Automation (ICMTMA), vol. 3, 2011, pp. 974-977.

[6] C. Vlachou, J. Herzen, and P. Thiran, "Fairness of MAC protocols: IEEE 1901 vs. 802.11," in Proceedings of the IEEE International Symposium on Power Line Communications and its Applications (ISPLC), 2013.

[7] G. Bianchi, "Performance Analysis of the IEEE 802.11 Distributed Coordination Function," IEEE Journal on Selected Areas in Coтmunications, vol. 18 , no. 3, pp. 535-547, 2000.

[8] P. Chatzimisios, A. C. Boucouvalas, and V. Vitsas, "Packet delay analysis of IEEE 802.11 MAC protocol," Electronics Letters, vol. 39, no. 18, pp $1358-1359,2003$

[9] M. Y. Chung, M.-H. Jung, T.-J. Lee, and Y. Lee, "Performance analysis of HomePlug 1.0 MAC with CSMA/CA," IEEE Journal on Selected Areas in Communications, vol. 24, no. 7, pp. 1411-1420, 2006.

[10] Y. Xiao, "A simple and effective priority scheme for IEEE 802.11," IEEE Communications Letters, vol. 7, no. 2, pp. 70-72, 2003.

[11] G. Chen, J. Branch, M. Pflug, L. Zhu, and B. Szymanski, "SENSE: A Sensor Network Simulator," Advances in Pervasive Computing and Networking, pp. 249-267, 2004. 